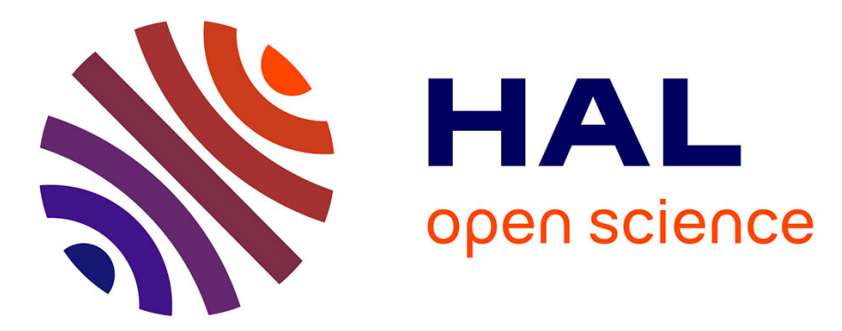

\title{
Renormalization of the fragmentation equation: Exact self-similar solutions and turbulent cascades
}

\author{
Vladimir Leonidovich Saveliev, Mikhael Gorokhovski
}

\section{To cite this version:}

Vladimir Leonidovich Saveliev, Mikhael Gorokhovski. Renormalization of the fragmentation equation: Exact self-similar solutions and turbulent cascades. Physical Review E : Statistical, Nonlinear, and Soft Matter Physics, 2012, 86, pp.061112. 10.1103/PhysRevE.86.061112 . hal-00780135

\section{HAL Id: hal-00780135 https://hal.science/hal-00780135}

Submitted on 11 Apr 2016

HAL is a multi-disciplinary open access archive for the deposit and dissemination of scientific research documents, whether they are published or not. The documents may come from teaching and research institutions in France or abroad, or from public or private research centers.
L'archive ouverte pluridisciplinaire HAL, est destinée au dépôt et à la diffusion de documents scientifiques de niveau recherche, publiés ou non, émanant des établissements d'enseignement et de recherche français ou étrangers, des laboratoires publics ou privés. 


\title{
Renormalization of the fragmentation equation: Exact self-similar solutions and turbulent cascades
}

\author{
V. L. Saveliev ${ }^{1,2}$ and M. A. Gorokhovski ${ }^{2}$ \\ ${ }^{1}$ Institute of Ionosphere, National Center of Space Research and Technology, 050020 Almaty, Kazakhstan \\ ${ }^{2}$ Laboratoire de Mécanique des Fluids et d'Acoustique, CNRS - Ecole Centrale de Lyon - INSA Lyon - Université Claude Bernard Lyon 1, \\ 36 Avenue Guy de Collongue, 69134 Ecully Cedex, France
}

(Received 22 June 2012; published 10 December 2012)

\begin{abstract}
Using an approach developed earlier for renormalization of the Boltzmann collision integral [Saveliev and Nanbu, Phys. Rev. E 65, 051205 (2002)], we derive an exact divergence form for the fragmentation operator. Then we reduce the fragmentation equation to the continuity equation in size space, with the flux given explicitly. This allows us to obtain self-similar solutions and to find the integral of motion for these solutions (we call it the bare flux). We show how these solutions can be applied as a description of cascade processes in three- and two-dimensional turbulence. We also suggested an empirical cascade model of impact fragmentation of brittle materials.
\end{abstract}

DOI: 10.1103/PhysRevE.86.061112

PACS number(s): 46.50.+a, 05.10.-a, 05.20.-y, 47.27.Gs

\section{INTRODUCTION}

The fragmentation process consists of the generation of random fragments (or particles) by successive breaking. The process occurs in numerous physical phenomena and engineering applications. Examples include rock fracture, degradation of large polymer chains, DNA fragmentation, cascade decay of turbulent eddies, droplet breakup, and decomposition of solid drugs in organisms. One approach that is often used to model these processes is the fragmentation under scaling symmetry model. The scaling symmetry means that at each fragmentation step, a parent particle splits into daughter particles with a partition probability independent of the particle size; i.e., the particle size $r$ is modified in a step-by-step manner by multiplication by a random multiplier $r \Rightarrow \alpha r ; 0 \leqslant \alpha \leqslant 1$. If the fragmentation rate is constant, then in the limit of a large number of breakups, the particle-size distribution $F(r, t)$ becomes lognormal, independent of the initial distribution. This universality was first explained by the Kolmogorov discrete model [1]. Later on, for fragmentation as a continuous process, Filippov [2] derived the linear integrodifferential equation that governs the temporal evolution of the particle-size distribution $F(r, t)$. This equation is called the fragmentation equation. There is a solid body of research concerning the analytical solutions to this equation with the fragmentation rate depending on $r$. In writing this equation for later use, we adopt the notation introduced in Ref. [3]:

$$
\frac{\partial F(r)}{\partial t}=\left(q_{0} \hat{I}_{+}^{p}-1\right) v(r) F(r) .
$$

Here $\hat{I}_{+}^{p}$ denotes the fragmentation operator,

$$
\begin{aligned}
\hat{I}_{+}^{p} F & =\int_{0}^{1} F\left(\frac{r}{\alpha}\right) Q(\alpha) \frac{d \alpha}{\alpha} \\
& =\int_{0}^{1} d \alpha Q(\alpha) \exp \left[-\ln \alpha\left(r \frac{\partial}{\partial r}+1\right)\right] F(r),
\end{aligned}
$$

and $v(r)$ is the fragmentation rate (or frequency), $q_{0}$ is the expected number of spawned particles from one split, and $Q(\alpha), \int_{0}^{1} Q(\alpha) d \alpha=1$, defines the probability density function of random multipliers $\alpha$ (hereafter this function is referred to as the fragmentation spectrum). Setting the mass of a particle to be proportional to the cube of its radius $\left(m \sim r^{3}\right)$, the mass-conservation condition yields $q_{0}=$ $1 /\left\langle\alpha^{3}\right\rangle_{Q},\left\langle\alpha^{3}\right\rangle_{Q}=\int_{0}^{1} \alpha^{3} Q(\alpha) d \alpha$. Here the choice of $m \sim r^{3}$ is not essential, and any other exponent can be employed in a similar way. The fragmentation events result in an increase in the total number of particles over time, while the total mass of particles is conserved. Therefore instead of the number distribution function $F(r)$, the mass distribution function $f(r)$ is usually used. The norm of this function is conserved. The two functions, $F(r)$ and $f(r)$, are related by a simple relation $f(r) \sim r^{3} F(r)$. Thus the fragmentation equation for $f(r)$ takes the following form:

$$
\frac{\partial f(r)}{\partial t}=\left(\hat{I}_{+}-1\right) v(r) f(r)
$$

where

$$
\begin{gathered}
\hat{I}_{+}=r^{3} q_{0} \hat{I}_{+}^{p} r^{-3}=\int_{0}^{1} d \alpha q(\alpha) \exp \left[-\ln \alpha\left(r \frac{\partial}{\partial r}+1\right)\right], \\
\int_{0}^{1} d \alpha q(\alpha)=1 \\
\exp \left[-\ln \alpha\left(r \frac{\partial}{\partial r}+1\right)\right] f(r)=\frac{1}{\alpha} f\left(\frac{r}{\alpha}\right) .
\end{gathered}
$$

The link between the two fragmentation spectra $q(\alpha)$ in Eq. (4) and $Q(\alpha)$ in Eq. (2) is $q(\alpha)=\alpha^{3} Q(\alpha) /\left\langle\alpha^{3}\right\rangle_{Q}$. Notice in particular that when the breakup frequency $v(r)$ is defined by a power law

$$
v(r)=c r^{\mu},
$$

the fragmentation equation has no sense for $\mu<0$ at $r=0$. However from physical intuition, zero-sized particles cannot undergo fragmentation, so it is natural to complete (3) with an additional condition for the fragmentation operator:

$$
\left(\hat{I}_{+}-1\right) \nu \delta(r)=0,
$$


which is equivalent to the following definition:

$$
v(r) \delta(r)=v(0) \delta(r), \quad v(0)=v_{0}<\infty .
$$

The first substantial progress in obtaining particular analytical solutions to the fragmentation equation was made in Refs. [2,4,5]. Among all the solutions, self-similar solutions are of special interest; these solutions are essentially the intermediate long-time asymptotics [2,6-8]. In these papers, the power function (5) for the fragmentation rate is used. It is worth noting that the solutions for the case of the constant rate, $\mu=0$, cannot be obtained from those solutions simply by taking the limit $\mu \rightarrow 0$. This case is analyzed in Ref. [3]. The emphasis is put on the existence of two successive intermediate asymptotics to Eq. (3) if $\mu=0$; these are lognormal and power-type asymptotics. For $\mu<0$, the interesting phenomenon of shattering transition ("condensation" to zero size) is described in Refs. [2,5,7,9]. We also refer to a more recent approach, in which the solutions to the fragmentation equation are constructed on the basis of continuous self-similar Markov processes [10,11]. Also different results concerning the existence and uniqueness of solutions to (3) are reported in Ref. [12]; see also the references therein.

The approach adopted in this paper is different from that employed in previous studies. Using scaling symmetry of the fragmentation operator, we obtain its new renormalized form by the method proposed in Ref. [13] for renormalization of the Boltzmann collision integral. In this way, the analytical subtraction of the loss term from the gain term appears in the fragmentation operator. Then we rewrite the fragmentation equation (3) as a continuity equation. The derived identity for the fragmentation operator allows us to reduce the integrodifferential equation to a pure differential equation in an exact way, and to obtain new, exact, self-similar solutions. Along with the continuity form of Eq. (3), a new controlling parameter for the self-similar solutions emerges naturally. This parameter controls the mass flux in size space; we call it the bare flux. Among new self-similar solutions, there are solutions with infinite norm $\int f(r) d r=\infty$, as well as solutions which are nonpositive. We suggest that there is a correspondence between the solutions obtained in this way and cascades of turbulent energy and vorticity moments, with emphasis on the corresponding to these physical values bare flux as the principal characteristic of these processes. In previous work we have applied symmetry methods in a group-theoretical description of turbulence [14], but on the basis of the Navier-Stokes equation, rather than in the framework of the empirico-mathematical model, as in the present paper. This empirico-mathematical model of turbulent cascade is based on the fragmentation equation (3). We also suggested an empirical cascade model of impact fragmentation of brittle materials.

\section{RENORMALIZATION OF THE FRAGMENTATION EQUATION}

Let us consider the fragmentation operator which, using Eq. (4), can be written as

$$
\hat{I}=\int_{0}^{1} d \alpha q(\alpha) e^{-(\ln \alpha) \hat{\sigma}}-1,
$$

where $\hat{\sigma}=\frac{\partial}{\partial r} r=r \frac{\partial}{\partial r}+1$. We expand the exponential operator $e^{-(\ln \alpha) \hat{\sigma}}$ as a Taylor series with residual term:

$$
\begin{aligned}
e^{(-\ln \alpha) \hat{\sigma}}= & 1+(-\ln \alpha) \hat{\sigma}+\frac{1}{2 !}(-\ln \alpha)^{2} \hat{\sigma}^{2} \\
& +\cdots+\frac{1}{(n-1) !}(-\ln \alpha)^{n-1} \hat{\sigma}^{n-1} \\
& +\frac{1}{n !}(-\ln \alpha)^{n} \hat{\sigma}^{n} \int_{0}^{1} d \beta p_{n}(\beta) e^{-\beta(\ln \alpha) \hat{\sigma}},
\end{aligned}
$$

where $n=1,2,3 \ldots$ and $p_{n}(\beta)=n(1-\beta)^{n-1}, \int_{0}^{1} d \beta p_{n}(\beta)=$ 1. Then introducing expansion (9) into the expression for the fragmentation operator (4), we obtain

$$
\begin{aligned}
\hat{I}_{+}= & 1+\langle-\ln \alpha\rangle \hat{\sigma}+\frac{1}{2 !}\left\langle(-\ln \alpha)^{2}\right\rangle \hat{\sigma}^{2} \\
& +\cdots+\frac{1}{(n-1) !}\left\langle(-\ln \alpha)^{n-1}\right\rangle \hat{\sigma}^{n-1} \\
& +\frac{1}{n !} \hat{\sigma}^{n} \int_{0}^{1} \int_{0}^{1} d \alpha d \beta q(\alpha)(-\ln \alpha)^{n} p_{n}(\beta) e^{-\beta(\ln \alpha) \hat{\sigma}}
\end{aligned}
$$

Introducing the change of variables $\alpha^{\prime}=\alpha^{\beta}, \beta^{\prime}=\alpha^{1-\beta}$ into the double integral in Eq. (10), for which the Jacobian of the transformation is equal to $\left(-\ln \alpha^{\prime} \beta^{\prime}\right)^{-1}$ and the inverse transformation is $\alpha=\alpha^{\prime} \beta^{\prime}, \beta=\ln \alpha^{\prime} / \ln \left(\alpha^{\prime} \beta^{\prime}\right)$, we have

$$
\begin{aligned}
\hat{I}_{+}= & +\langle-\ln \alpha\rangle \hat{\sigma}+\frac{\left\langle(-\ln \alpha)^{2}\right\rangle}{2 !} \hat{\sigma}^{2} \\
& +\cdots+\frac{\left\langle(-\ln \alpha)^{n-1}\right\rangle}{(n-1) !} \hat{\sigma}^{n-1}+\frac{\left\langle(-\ln \alpha)^{n}\right\rangle}{n !} \hat{\sigma}^{n} \hat{I}_{+}^{(n)},
\end{aligned}
$$

where $\left\langle(-\ln \alpha)^{n}\right\rangle=\int_{0}^{1}(-\ln \alpha)^{n} q(\alpha) d \alpha$ and

$$
\hat{I}_{+}^{(n)}=\int_{0}^{1} d \alpha q_{n}(\alpha) e^{-(\ln \alpha) \hat{\sigma}} .
$$

Notice that operator $\hat{I}_{+}^{(n)}$ has the same form as the operator $\hat{I}_{+}$, but with the new fragmentation spectrum $q_{n}(\alpha)$ :

$$
\begin{aligned}
q_{n}(\alpha) & =\frac{n}{\left\langle(-\ln \alpha)^{n}\right\rangle} \int_{0}^{1} q(\alpha \beta)(-\ln \beta)^{n-1} d \beta, \\
\int_{0}^{1} q_{n}(\alpha) d \alpha & =1 .
\end{aligned}
$$

The inverse formula can be obtained by differentiating Eq. (13) with respect to $\alpha$ :

$$
\frac{\left\langle(-\ln \alpha)^{n}\right\rangle}{n !}\left(\frac{\partial}{\partial \alpha} \alpha\right)^{n} q_{n}(\alpha)=q(\alpha) .
$$

The representation of the operator $\hat{I}_{+}$by Eq. (11) allows us to rewrite the fragmentation equation in the form of the continuity equation:

$$
\frac{\partial f(r)}{\partial t}=-\frac{\partial}{\partial r} j,
$$


where the mass flux in size space is given by

$$
\begin{aligned}
j= & -r\left[\langle-\ln \alpha\rangle+\frac{\left\langle(-\ln \alpha)^{2}\right\rangle}{2 !} \hat{\sigma} \cdots+\frac{\left\langle(-\ln \alpha)^{n-1}\right\rangle}{(n-1) !} \hat{\sigma}^{n-2}\right. \\
& \left.+\frac{\left\langle(-\ln \alpha)^{n}\right\rangle}{n !} \hat{\sigma}^{n-1} \hat{I}_{+}^{(n)}\right] \nu(r) f(r) .
\end{aligned}
$$

The number of terms in Eq. (16) can be chosen arbitrarily $(n=1,2,3 \ldots)$ until the moments $\left\langle(-\ln \alpha)^{n}\right\rangle$ remain finite. It can be seen from the continuity equation (15) that each particle (a so-called quasiparticle) moves at a velocity $v=j / f(r, t)$ along the half line $0 \leqslant r<\infty$. The value of the velocity is defined by the distribution function $f(r, t)$, i.e., by the positions of all the other quasiparticles. The primary fragmentation equation (3) can also be interpreted as describing the evolution of a system of particles, but these particles "jump" on the $r$ half line; such particles have no velocity. The transition from Eqs. (3) to (15) illustrates an important physical statement [13]: When a system of particles is described statistically by a distribution function, the microdynamics of such a system can be substantially modified, but the distribution function will remain unchanged. Notice also that Eqs. (15) and (16) define explicitly the mass flux carried in size space during the fragmentation process.

For the practically important cases $n=1$ and $n=2$, Eqs. (15) and (16) yield two simple corresponding equations:

$$
\begin{gathered}
\frac{\partial}{\partial t} f=\frac{\partial}{\partial r} r\langle-\ln \alpha\rangle \hat{I}_{+}^{(1)} v f, \\
q_{1}(\alpha)=\frac{1}{\langle-\ln \alpha\rangle} \int_{0}^{1} q(\alpha \beta) d \beta ; \\
\frac{\partial}{\partial t} f=\frac{\partial}{\partial r} r\left(\langle-\ln \alpha\rangle+\frac{1}{2}\left\langle(\ln \alpha)^{2}\right\rangle \frac{\partial}{\partial r} r \hat{I}_{+}^{(2)}\right) v f, \\
q_{2}(\alpha)=\frac{2}{\left\langle(\ln \alpha)^{2}\right\rangle} \int_{0}^{1} q(\alpha \beta)(-\ln \beta) d \beta .
\end{gathered}
$$

If the fragmentation spectrum $q(\alpha)$ is a power function $q(\alpha)=$ $(\gamma+1) \alpha^{\gamma}$ with $\gamma>-1$, then expressions (12), (13) can be simplified significantly:

$$
q_{n}(\alpha)=q(\alpha), \quad \hat{I}_{+}^{(n)}=\hat{I}_{+}, \quad\left\langle(-\ln \alpha)^{n}\right\rangle=\frac{n !}{(\gamma+1)^{n}} .
$$

It should be noticed that in this case, the operators $\hat{I}_{+}^{(n)}$ and $\hat{I}_{+}$are equal, and expansion (11) reduces to the identity for operator $\hat{I}_{+}$:

$$
\begin{aligned}
\hat{I}_{+}= & +\frac{\hat{\sigma}}{(\gamma+1)}+\frac{\hat{\sigma}^{2}}{(\gamma+1)^{2}} \\
& +\cdots+\frac{\hat{\sigma}^{n-1}}{(\gamma+1)^{n-1}}+\frac{\hat{\sigma}^{n}}{(\gamma+1)^{n}} \hat{I}_{+} .
\end{aligned}
$$

If $n=1$, this identity leads to the useful equation

$$
\left[1-\frac{1}{(\gamma+1)} \frac{\partial}{\partial r} r\right] \hat{I}_{+}=1 .
$$

Finally notice that $\hat{I}_{+} v(r) f(r)$ in Eq. (3) will converge if at infinity the distribution function $f(r)$ decreases faster than $r^{(\gamma-\mu)}$ :

$$
\frac{f(r)}{r^{(\gamma-\mu)}} \underset{r \rightarrow \infty}{\rightarrow} 0
$$

\section{INTERMEDIATE ASYMPTOTICS: SELF-SIMILAR SOLUTIONS TO THE RENORMALIZED FRAGMENTATION EQUATION}

Let us consider the renormalized $(n=1)$ fragmentation equation (17):

$$
\frac{\partial}{\partial t} f=-\frac{\partial}{\partial r} j, \quad j=-r\langle-\ln \alpha\rangle \hat{I}_{+}^{(1)} \nu f .
$$

We have $\hat{I}_{+}^{(1)} \frac{1}{r}=\frac{1}{r}, \hat{I}_{+}^{(1)} \delta(r)=\delta(r)$, and then one exact solution to Eq. (23) is given by

$$
\begin{aligned}
f(r, t) & =-j_{0}\left(\frac{1}{\langle-\ln \alpha\rangle \nu r}+t \delta(r)\right), \\
j_{0} & <0, \quad 0 \leqslant r<\infty .
\end{aligned}
$$

The mass flux corresponding to this solution is a constant, denoted by $j_{0}$ :

$$
j=-r\langle-\ln \alpha\rangle \hat{I}_{+}^{(1)} \nu f=j_{0}, \quad j_{0}<0 .
$$

So, solution (24) generates a stationary mass flux $j_{0}$ in size space during the fragmentation process. Since $j_{0}<0$, the mass is carried from infinity towards zero size, and there is therefore an accumulation of zero-sized particles. If the fragmentation rate $v(r)$ is a power function (5), the velocity of motion of a quasiparticle along the $r$ half line reads

$$
v=\frac{j_{0}}{f(r)}=-\langle-\ln \alpha\rangle c r^{(1+\mu)}, \quad 0<r<\infty .
$$

If $\mu>-1$, the quasiparticles move towards zero size with a velocity that decreases towards zero, while their mass density grows in such a way that the mass flux remains constant. If $\mu=-1$, quasiparticles are uniformly distributed on the size line and quasiparticles move towards zero size at constant velocity. If $\mu<-1$, the quasiparticles move towards zero size with an increasing velocity.

The next step is to construct exact self-similar solutions to the fragmentation equation (23). The significance of this step is that the self-similar solutions are the long-time intermediate asymptotics for solutions to the fragmentation equation. In order to obtain these solutions, we define a scaling transformation $\hat{S}$ with parameter $|\bar{t}|^{\mu^{-1}}$ :

$$
f(r, t)=\hat{S} \phi(r, t)=|\bar{t}|^{\mu^{-1}} \phi\left(|\bar{t}|^{\mu^{-1}} r, t\right)=e^{\mu^{-1} \ln |\bar{t}| \hat{\sigma}} \phi(r, t),
$$

for which the inverse transformation is

$$
\begin{aligned}
\phi(r, t) & =\hat{S}^{-1} f(r, t)=\frac{1}{|\bar{t}|^{\mu^{-1}}} f\left(\frac{r}{|\bar{t}|^{\mu^{-1}}}, t\right) \\
& =e^{-\mu^{-1} \ln |\bar{t}| \hat{\sigma}} f(r, t) .
\end{aligned}
$$

The parameter $\bar{t}$ is considered to be a linear function of time $\bar{t}=\tau_{0}^{-1} t$, where $\tau_{0}$ is a positive constant. Under these transformations, the norm of the distribution function (the total mass of particles in the system) is conserved $\int d r f(r, t)=$ $\int d r \phi(r, t)$, provided that it exists. Obviously, at $t=\tau_{0}$, the two distribution functions $\phi$ and $f$ are equal $\phi\left(r, \tau_{0}\right)=f\left(r, \tau_{0}\right)$. Under scaling transformations (27) and (28), the operators in 
Eq. (23), because of $\frac{\partial \ln |\bar{t}|}{\partial \bar{t}}=\frac{1}{\bar{t}}$, are transformed as follows:

$$
\begin{aligned}
& \hat{S}^{-1} \hat{I}_{+}^{(1)} \hat{S}=\hat{I}_{+}^{(1)}, \quad \hat{S}^{-1} \hat{\sigma} \hat{S}=\hat{\sigma}, \\
& \hat{S}^{-1} v(r) \hat{S}=\frac{1}{|\bar{t}|} v(r), \quad \hat{S}^{-1} \frac{\partial}{\partial t} \hat{S}=\frac{\partial}{\partial t}+\frac{1}{\mu \tau_{0} \bar{t}} \hat{\sigma} .
\end{aligned}
$$

Applying the inverse scaling transformation operator $\hat{S}^{-1}$ to Eq. (23) and taking into account Eq. (29), we obtain the equation for $\phi(r, t)$ :

$$
\frac{\partial \phi(r, t)}{\partial t}=\frac{1}{|\bar{t}|} \frac{\partial}{\partial r} r\left[-\frac{|\bar{t}|}{\bar{t}} \frac{1}{\mu \tau_{0}}+\langle-\ln \alpha\rangle \hat{I}_{+}^{(1)} v\right] \phi .
$$

Here it is convenient to introduce the staircase function $\tau(t)$ :

$$
\tau(t)=\frac{\bar{t}}{|\bar{t}|} \tau_{0}=\left\{\begin{array}{cc}
\tau_{0}, & t>0, \\
-\tau_{0}, & t<0 .
\end{array}\right.
$$

Then Eq. (30) can finally be rewritten as

$$
\frac{\partial \phi(r, t)}{\partial t}=\frac{1}{|\bar{t}|} \frac{\partial}{\partial r} r\left[-\frac{1}{\mu \tau(t)}+\langle-\ln \alpha\rangle \hat{I}_{+}^{(1)} v\right] \phi, \quad \tau^{-1} t>0 .
$$

There are then two different cases: $\tau>0$ and $\tau<0$. If $\tau>0$, Eq. (32) describes a fragmentation process which continues for an arbitrary long period $(0<t<\infty)$. On the other hand, if $\tau<0$, Eq. (32) describes a fragmentation process which terminates at $t=0(-\infty<t<0)$. From the definition of $\tau(t)$, $\tau^{-1} t>0$ for both cases. It is seen that Eq. (32) describes the relaxation of the general solution $\phi(r, t)$ to the stationary one $\phi(r)$, satisfying the following equation:

$$
\frac{\partial}{\partial r} r\left[-\frac{1}{\mu \tau}+\langle-\ln \alpha\rangle \hat{I}_{+}^{(1)} v\right] \phi(r)=0 .
$$

The intermediate asymptotics to the fragmentation equation (23) can be obtained from $\phi(r)$ using the scaling transformation (27). From Eq. (33), we have

$$
r\left[\frac{1}{\mu \tau}-\langle-\ln \alpha\rangle \hat{I}_{+}^{(1)} v\right] \phi=j_{0} .
$$

Here again $j_{0}$ is a constant - the bare mass flux-and the total mass flux given by Eq. (23) can be expressed in terms of this new integral of motion. Dividing Eq. (34) by $r$ and applying the scaling transformation $\hat{S}$, making use of the property $\hat{S} r^{-1}=$ $r^{-1}$, one gets

$$
j=\frac{\tau}{t}\left(j_{0}-\frac{r}{\mu \tau} f\right) .
$$

The exact solution to Eq. (34) can be readily obtained for the power spectrum: $q(\alpha)=(\gamma+1) \alpha^{\gamma}$. For this spectrum $\hat{I}_{+}^{(1)}=$ $\hat{I}_{+},\langle-\ln \alpha\rangle=(\gamma+1)^{-1}$, and from Eq. (21), the operator $\hat{I}_{+}$satisfies the following relation: $(\gamma+1-\hat{\sigma}) \hat{I}_{+}=\gamma+1$. Dividing Eq. (34) by $r$, and then applying the operator $1+\gamma-\hat{\sigma}$, we obtain the differential equation for $\phi(r)$ :

$$
\left[r \frac{\partial}{\partial r}-\gamma+\mu \tau \nu\right] \phi(r)=-\frac{(1+\gamma) \mu \tau}{r} j_{0} .
$$

The eigenvalue of the operator $1+\gamma-\hat{\sigma}$ is zero for the eigenfunction $r^{\gamma}$ :

$$
(1+\gamma-\hat{\sigma}) r^{\gamma}=0 .
$$

Therefore, in addition to the solutions to Eq. (34), Eq. (36) also possesses a spurious solution, given by the solution to Eq. (34) with the function const $\times r^{\gamma+1}$ added to its right-hand side. Notice that for large $r$, such solutions behave as $\sim r^{\gamma-\mu}$ if $\mu>0$, or as $\sim r^{\gamma}$ if $\mu<0$. We will use this asymptotic behavior to exclude the spurious solutions. Equation (36) is a first-order linear differential equation for which the solutions are well known. If the right-hand side of Eq. (36) is equal to zero $\left(j_{0}=0\right)$, this equation becomes

$$
\left[r \frac{\partial}{\partial r}-\gamma+\mu \tau \nu\right] \phi_{1}(r)=0, \quad j_{0}=0 .
$$

The solution to this equation is

$$
\phi_{1}(r)=\frac{\text { const }}{r}\left(\frac{\nu}{c}\right)^{-\alpha} e^{-\tau \nu}=\text { const }_{1} r^{\gamma} e^{-\tau c r^{\mu}},
$$

where $\alpha=-\frac{1+\gamma}{\mu}$. When the right-hand side of Eq. (36) is nonzero, $j_{0} \neq 0$, the solution can be expressed in terms of $\phi_{1}(r)$ :

$$
\phi_{2}(r)=-(1+\gamma) \mu \tau j_{0} \phi_{1}(r) \int_{r_{0}}^{r} \phi_{1}^{-1}(r) \frac{d r}{r^{2}},
$$

where $r_{0}$ is a constant. Inserting $v=c r^{\mu}$ into Eq. (40) leads to a standard integral:

$$
\begin{aligned}
\phi_{2}(r) & =-\frac{(1+\gamma) j_{0} \tau}{r} v^{-\alpha} e^{-\tau \nu} \int_{\nu_{0}}^{\nu} x^{\alpha-1} e^{\tau x} d x, \\
\alpha & =-\frac{1+\gamma}{\mu}, \quad v=c r^{\mu} .
\end{aligned}
$$

The function $\phi_{2}(r)$ changes sign, when $r=r_{0}, v\left(r_{0}\right)=v_{0}$. So, if we require $\phi_{2}(r)$ to be positive over the interval $0<r<$ $\infty(0<v<\infty)$, then the constant $v_{0}$ should be taken equal to either zero $\left(v_{0}=0\right)$ or infinity $\left(v_{0}=\infty\right)$. Assuming $v_{0}$ takes one of these two values, we then have four different cases, according to the combination of the signs of the parameters $\mu$ and $\tau$, subject to the constraint that the exponents $\mu$ and $\alpha$ must have opposite signs, since $\gamma+1$ is always positive. The four different cases are represented in Table I.

Only some of these combinations are possible: In the first case, solution $\phi_{2}(r)$ must be discarded; in the second case, only $v_{0}=\infty$ is possible; and in the third case, only $v_{0}=0$ is possible. In the fourth case, the integral is convergent for both values of $v_{0}\left(v_{0}=0\right.$ and $\left.v_{0}=\infty\right)$. However as will be seen later, the choice $v_{0}=\infty$ gives a spurious solution. The integral in Eq. (41) can be expressed by confluent hypergeometric functions [15] $\Phi(1,1+\alpha ; x)$ and $\Psi(1,1+\alpha ; x)$; if $\alpha$ is an integer, then $\Phi(1,1+\alpha ; x)$ is the elementary function (see Appendix).

In the fragmentation process, we have essentially three different cases with respect to the fragmentation rate dependency on $r$ : the exponent $\mu$ can be positive, negative, or equal to zero $(\mu>0, \mu<0, \mu=0)$. Notice in addition that Eq. (36) 
TABLE I. Choice of positive solution.

\begin{tabular}{|c|c|c|c|c|c|}
\hline No. & Sign $\mu$ & Sign $\tau$ & $\begin{array}{l}\text { Applicability of } \\
\text { solution } \phi_{1}\end{array}$ & $\begin{array}{c}\text { Convergence of } \\
\text { integral at } \nu_{0}=0 ; \\
\text { applicability of solution } \phi_{2}\end{array}$ & $\begin{array}{c}\text { Convergence of } \\
\text { integral at } v_{0}=\infty ; \\
\text { applicability of solution } \phi_{2}\end{array}$ \\
\hline 1 & $\mu>0$ & $\tau>0$ & yes & integral diverges & integral diverges \\
\hline 2 & $\mu>0$ & $\tau<0$ & no & integral diverges & integral converges; $\phi_{2}$ suitable \\
\hline 3 & $\mu<0$ & $\tau>0$ & no & integral converges; $\phi_{2}$ suitable & integral diverges \\
\hline 4 & $\mu<0$ & $\tau<0$ & no & integral converges; $\phi_{2}$ suitable & integral converges; $\phi_{2}$ not suitable \\
\hline
\end{tabular}

describes a relaxation process which can either continue indefinitely in time (if $\tau>0$ ) or, if $\tau<0$, will terminate at $t=0$. We consider these cases separately.

\section{A. Case $0<\mu$ : Decreasing breakup frequency with decreasing fragment size}

\section{Staircase function $\tau$ is greater than zero, $0<\tau$}

In this case, the fragmentation process will continue indefinitely $(0<t<\infty)$, and the first solution (39) to Eq. (33) satisfies the applicability requirements (see Table I):

$$
\begin{aligned}
\phi_{1}(r) & =\text { const }_{1} r^{\gamma} e^{-\tau c r^{\mu}}, \quad 0<\mu, \\
0 & <\tau, \quad 0<t<\infty .
\end{aligned}
$$

This can be readily verified by direct substitution of Eq. (42) into Eq. (33). According to Eq. (27), solution (42) corresponds to the following self-similar solution to both the fragmentation equation (3) and the renormalized equation (17):

$$
\begin{aligned}
f_{1}(r, t) & =\operatorname{const}_{1}\left(\tau_{0}^{-1} t\right)^{(\gamma+1) / \mu} r^{\gamma} e^{-t c r^{\mu},} \\
0 & <\mu, \quad 0<t<\infty .
\end{aligned}
$$

The corresponding mass flux can be obtained from Eq. (35):

$$
\begin{aligned}
& j(r, t)=\frac{\text { const }_{1}}{\mu \tau_{0}}\left(\tau_{0}^{-1} t\right)^{(\gamma-\mu+1) / \mu} r^{\gamma+1} e^{-t c r^{\mu}}, \\
& j \underset{r \rightarrow 0}{\rightarrow} 0, \quad j \underset{r \rightarrow \infty}{\rightarrow} 0 .
\end{aligned}
$$

This mass flux tends to zero as the size goes to zero $(r=0)$. Hence there is no accumulation of zero-sized particles in this case. As the size increases to infinity, this flux also tends to zero (no particles entering from infinity). The second-type positive solution $\phi_{2}(r)$ with $\mu>0, \tau>0$ does not exist, since the integral (41) is divergent for both $v_{0}=0$ and $v_{0}=\infty$.

\section{Staircase function $\tau$ is less than zero, $\tau<0$}

The fragmentation process starts at some arbitrary negative time and is completed at zero time $(-\infty<t<0)$. Generally speaking, in this case the first-type solution $\phi_{1}(r)$ has no obvious meaning, since the integral in the fragmentation operator is divergent:

$$
\begin{aligned}
\hat{I}_{+} \nu \phi_{1} & =\int_{0}^{1} d \alpha(\gamma+1) \alpha^{\gamma} \frac{1}{\alpha} c\left(\frac{r}{\alpha}\right)^{\mu} \phi_{1}\left(\frac{r}{\alpha}\right)=\infty, \\
0 & <\mu, \quad \tau<0 .
\end{aligned}
$$

Consider now the applicability of the second-type solution $\phi_{2}(r)$. If $\tau<0$ and $v_{0}=\infty$, the integral in Eq. (41) is convergent. This implies the existence of the second-type positive solution (41) to Eq. (36). This solution will describe the fragmentation process which completes as time approaches zero:

$$
\begin{aligned}
& 0<\mu, \quad \tau<0, \quad-\infty<t<0, \\
& \phi_{2}(r)=-\frac{(1+\gamma) j_{0} \tau}{r} v^{-\alpha} e^{-\tau \nu} \int_{\infty}^{\nu} x^{\alpha-1} e^{\tau x} d x \\
&=\frac{(1+\gamma) j_{0} \tau}{r} \Psi(1,1+\alpha,-\tau \nu), \\
& \phi_{2}(r) \underset{r \rightarrow 0}{\sim} \frac{j_{0} \mu \tau}{r}, \quad \phi_{2}(r) \underset{r \rightarrow \infty}{\sim}-\frac{(1+\gamma) j_{0}}{r v},
\end{aligned}
$$

where $\alpha=-\frac{1+\gamma}{\mu}, v=c r^{\mu}, j_{0}<0$.

The self-similar solution to the equivalent primary equations (3) and (17) can then be obtained from Eq. (46) together with Eq. (27):

$$
\begin{aligned}
0 & <\mu, \quad \tau<0, \quad-\infty<t<0, \\
f_{2}(r, t) & =\frac{(1+\gamma) j_{0} \tau}{r} \Psi(1,1+\alpha,-v t), \quad \alpha<0, \quad(47) \\
j_{0} & <0, \quad f_{2}(r, t) \underset{r \rightarrow 0}{\sim} \frac{j_{0} \mu \tau}{r}, \quad f_{2}(r, t) \underset{r \rightarrow \infty}{\sim}-\frac{(1+\gamma) j_{0} \tau}{r \nu t} .
\end{aligned}
$$

According to Eq. (35), this solution yields the following mass flux:

$$
\begin{aligned}
& j(r, t)=\frac{j_{0} \tau}{t}\left[1-\frac{(1+\gamma)}{\mu} \Psi(1,1+\alpha,-v t)\right], \\
& j \rightarrow \underset{r \rightarrow 0}{\rightarrow} \frac{\tau}{t}\left(j_{0}-j_{0}\right)=0, \\
& j \underset{r \rightarrow \infty}{\sim} \frac{j_{0} \tau}{t}\left[1+\frac{(1+\gamma)}{\mu \nu t}\right] \underset{t \rightarrow-\infty}{\rightarrow} \frac{j_{0} \tau}{t}, \\
& \alpha<0, \quad j_{0}<0 .
\end{aligned}
$$

As the size $r$ decreases to zero, the mass flux goes to zero (no accumulation of zero-sized particles), and as the size increases towards infinity, the mass flux becomes independent of size, and is negative. This implies that particles enter from infinity, and when the time approaches zero, the mass flux from infinity increases to infinity.

We conclude that in the case of $0<\mu$, there are two suitable solutions: Eq. (47), which is applicable to the negative time interval $-\infty<t<0$, and solution (43), which is applicable for $0<t<\infty$. These two solutions can be combined to cover 
the complete time domain:

$$
\begin{aligned}
& 0<\mu, \quad \alpha<0, \quad j_{0}<0, \\
& f(r, t)= \begin{cases}-\frac{(1+\gamma) j_{0} \tau_{0}}{r} \Psi\left(1,1+\alpha,-t c r^{\mu}\right), & -\infty<t<0, \\
\operatorname{const}_{1}\left(\tau_{0}^{-1} t\right)^{-\alpha} r^{\gamma} e^{-t c r^{\mu},} & 0<t<\infty,\end{cases} \\
& f(r, t) \underset{t \rightarrow-\infty}{\sim} \frac{(1+\gamma) j_{0} \tau_{0}}{r \nu t}, \quad f(r, t) \underset{t \rightarrow-0}{\sim}-\frac{j_{0} \mu \tau_{0}}{r}, \\
& \alpha=-\frac{\gamma+1}{\mu} .
\end{aligned}
$$

The asymptotic behavior of solution (49) for small and large sizes is given in the Appendix.

As can be seen from Eq. (49), when time goes to minus infinity, $f(r, t)$ tends to the stationary solution (24) for $0<r<$ $\infty$. This explains why the stationary solution (24) is unstable with respect to the solution in Eq. (49). As time approaches zero, this solution provides the mass distribution with behavior as $\sim r^{-1}$. This implies an absorption of an infinite amount of mass from infinitely large sizes.

\section{B. Case $\mu<0$ : Increase of the fragmentation rate with decreasing size of fragments}

\section{Staircase function $\tau$ is greater than zero, $0<\tau$}

For large sizes, the first-type solution $\phi_{1}(r)$ behaves as $\sim r^{\gamma}$, for any set of parameters in the region $\mu<0$ and $-1<\gamma$. Then it follows from the preceding remarks on the asymptotic behavior of spurious solutions that $\phi_{1}(r)$ with $\mu<0$ is a spurious solution, and it will not be considered further.

For the second-type solution $\phi_{2}(r)$ for positive values of $\tau>0$, the integral in Eq. (41) becomes convergent at $v_{0}=0$, and the second-type positive solution to Eq. (36) then reads as follows:

$$
\begin{aligned}
& \mu<0, \quad 0<\tau, \quad 0<t<\infty, \\
& \phi_{2}(r)=-\frac{(1+\gamma) j_{0} \tau}{r} v^{-\alpha} e^{-\tau \nu} \int_{0}^{\nu} x^{\alpha-1} e^{\tau x} d x \\
&=-\frac{(1+\gamma) j_{0} \tau}{r} \alpha^{-1} \Phi(1,1+\alpha,-\tau \nu), \\
& \phi_{2}(r) \underset{r \rightarrow 0}{\sim}-\frac{(1+\gamma) j_{0}}{v r}, \quad \phi_{2}(r) \underset{r \rightarrow \infty}{\sim} \frac{\mu j_{0} \tau}{r} .
\end{aligned}
$$

Finally, from Eq. (27), the self-similar solution to Eqs. (3) and (17), corresponding to (50), is

$$
\begin{aligned}
\mu & <0, \quad 0<\tau, \quad 0<t<\infty, \\
f_{2}(r, t) & =-\frac{(1+\gamma) j_{0} \tau}{r} \alpha^{-1} \Phi(1,1+\alpha,-v t), \\
f_{2}(r, t) & \sim \underset{r \rightarrow 0}{\sim}-\frac{(1+\gamma) j_{0} \tau}{r \nu t}, \quad f_{2}(r, t) \underset{r \rightarrow \infty}{\sim} \frac{\mu j_{0} \tau}{r},
\end{aligned}
$$

with the mass flux

$$
\begin{aligned}
& j=\frac{j_{0} \tau}{t}[1-\Phi(1,1+\alpha,-v t)], \\
& j<0, \quad j \underset{r \rightarrow 0}{\sim} \frac{j_{0} \tau}{t}\left(1-\frac{\alpha}{v t}\right) \underset{r \rightarrow 0}{\rightarrow} \frac{j_{0} \tau}{t}, \\
& j \underset{r \rightarrow \infty}{\sim} j_{0} \frac{v \tau}{1+\alpha}\left(1-\frac{v t}{(2+\alpha)}\right) \underset{r \rightarrow \infty}{\rightarrow} 0 .
\end{aligned}
$$

Here the mass flux is negative and is directed towards zero size. It has a nonzero limiting value at zero size. This provides an accumulation of mass in a system of particles with zero size. With increasing size $r$, the mass flux $j(r, t)$ goes to zero and there is no mass entering from infinity. At the initial time, the mass flux to zero size is infinite, and then, as time progresses, this flux decreases to zero.

\section{Staircase function $\tau$ is less than zero, $\tau<0$}

The fragmentation process is completed at zero time, $-\infty<t<0$. For large sizes, the first-type solution $\phi_{1}(r)$ behaves as $\sim r^{\gamma}$. Once again, the asymptotic behavior of the solution indicates that it is a spurious solution, as discussed above, and should therefore be neglected.

The second-type solution $\phi_{2}(r)$ for $\mu<0$ and $\tau<0$ is meaningful for both $v_{0}=0$ and $v_{0}=\infty$. For $v_{0}=\infty$ this solution is

$$
\begin{aligned}
\mu & <0, \quad \tau<0, \\
\phi_{2}(r) & =\frac{(1+\gamma) j_{0} \tau}{r} v^{-\alpha} e^{-\tau \nu} \int_{\nu}^{\infty} x^{\alpha-1} e^{\tau x} d x \\
& =\frac{(1+\gamma) j_{0} \tau}{r} \Psi(1,1+\alpha,-\tau \nu) .
\end{aligned}
$$

But, when the size tends to infinity, solution (53) grows as $r^{\gamma}$, and should then be neglected. For $\nu_{0}=0$, the solution $\phi_{2}(r)$ is

$$
\begin{aligned}
\mu & <0, \quad \tau<0, \\
\phi_{2}(r) & =-\frac{(1+\gamma) j_{0} \tau}{r} v^{-\alpha} e^{-\tau \nu} \int_{0}^{\nu} x^{\alpha-1} e^{\tau x} d x \\
& =-\frac{(1+\gamma) j_{0} \tau}{r} \alpha^{-1} \Phi(1,1+\alpha,-\tau \nu) .
\end{aligned}
$$

This solution has the following asymptotic behavior:

$$
\begin{aligned}
\phi_{2}(r) \underset{r \rightarrow 0}{\sim}-\frac{(1+\gamma) j_{0} \tau}{r}(-\tau \nu)^{-\alpha} e^{-\tau \nu} \\
=-\frac{(1+\gamma) j_{0} \tau}{r}(-\tau c)^{(1+\gamma) / \mu} r^{\gamma+1} e^{-\tau c r^{\mu}}, \\
\phi_{2}(r) \underset{r \rightarrow \infty}{\sim} \frac{\mu j_{0} \tau}{r}, \quad j_{0}>0 .
\end{aligned}
$$

Note that here the bare mass flux $j_{0}$ is positive, $j_{0}>0$. This solution is physically possible. Using Eq. (27), the corresponding solution to Eqs. (3) and (17) is given by

$$
\begin{aligned}
\mu & <0, \quad \tau<0, \quad-\infty<t<0, \\
f_{2}(r, t) & =-\frac{(1+\gamma) j_{0} \tau}{r} \alpha^{-1} \Phi(1,1+\alpha,-t v), \\
\nu & =c r^{\mu}, \quad 0<j_{0} .
\end{aligned}
$$

From (35), the corresponding mass flux for this solution is

$$
\begin{aligned}
& j=\frac{j_{0} \tau}{t}[1-\Phi(1,1+\alpha,-t v)], \\
& j \underset{r \rightarrow 0}{\sim}-\frac{j_{0} \tau}{t} \alpha(-t \nu)^{-\alpha} e^{-t v} \underset{t \rightarrow 0}{\rightarrow}-\infty, \quad j \underset{r \rightarrow \infty}{\rightarrow} 0 .
\end{aligned}
$$

It is worth noting that in this case, the mass flux to zero size is infinite. This is consistent with the fact that the fragmentation process has to be completed over a finite time. As the size increases towards infinity, the flux tends to zero. Thus for negative values of $\mu$, the solution for negative times is given 
by expression (56), whereas for positive times, the solution is determined by expression (50). Finally, these two solutions can be combined to give

$$
\begin{aligned}
& \mu<0, \quad-\infty<t<\infty, \\
& f(r, t)=\frac{(1+\gamma)\left|j_{0}\right| \tau_{0}}{r} \alpha^{-1} \Phi(1,1+\alpha,-v t), \\
& \nu=c r^{\mu}, \quad j=-\frac{\left|j_{0}\right| \tau_{0}}{t}[1-\Phi(1,1+\alpha,-v t)], \\
& j<0, \quad j(r=0)=-\frac{\left|j_{0}\right| \tau_{0}}{t}, \quad 0<t<\infty .
\end{aligned}
$$

The asymptotic behavior of solution (58) in time and in size is given in the Appendix.

The solution (58) describes the fragmentation process, subjected to a second-order phase transition at zero time, $t=0$. As time advances from minus to plus infinity, the distribution function evolves continuously. For negative times $t<0$, small scales are dominant, and the mass distribution grows exponentially as the size decreases. The mass flux at zero size is infinite. When the time reaches zero, a discontinuity occurs: The mass flux "jumps" from an infinite value for negative times to a finite one for positive times, and then the flux decreases inversely with time. It is worth emphasizing the universal character of the mass-flux behavior with time. As the size tends to zero, for all possible values of the fragmentation parameters $\gamma, \mu, j_{0}, \tau_{0}$, and finite mass flux, this flux decreases inversely with time. Once the second-order phase transition takes place, two constants, $j_{0}$ and $\tau$, change sign. At the instant of the phase transition, the mass is distributed inversely with size:

$$
f(r, t=0)=\frac{-\mu\left|j_{0}\right| \tau_{0}}{r}
$$

\section{Application to turbulent cascade}

Solution (58) has an interesting application to the description of the turbulent cascade process. In inviscid flows, mechanical energy is conserved, so instead of mass distribution in size space, we could consider the distribution of specific turbulent energy in the size space of turbulent eddies undergoing a turbulent cascade. The specific energy distribution function and the specific energy flux have the following dimensions: $[f]=\frac{v^{2}}{r}$ and $[j]=\frac{v^{2}}{r} v=\frac{r^{2}}{t^{3}}$, respectively. The eddy-fragmentation process, described by solution (58), is characterized by two dimensional constants $j_{0}$ and $c$. For maximum symmetry of this solution, we assume that constant $c$ can be expressed in terms of $j_{0}$ :

$$
c=\left|j_{0}\right|^{x} .
$$

Dimensional analysis yields:

$$
\left[\frac{r^{-\mu}}{t}\right]=\left[\frac{r^{2}}{t^{3}}\right]^{x} .
$$

This gives

$$
x=\frac{1}{3}, \quad \mu=-\frac{2}{3}, \quad \alpha=\frac{3}{2}(1+\gamma) .
$$

In infinitely high Reynolds number turbulence, the flux of specific turbulent energy at zero size has the standard notation $\varepsilon$, and is known as the rate of turbulent energy dissipation per unit mass. According to Eq. (A6) for positive times, this dissipation rate is

$$
\varepsilon=-j(r=0)=\frac{\left|j_{0}\right| \tau_{0}}{t}, \quad 0<t<\infty .
$$

Otherwise, according to Eq. (A6) when time takes nonpositive values, the turbulent dissipation is infinite:

$$
\varepsilon=-j(r=0)=\infty, \quad-\infty<t \leqslant 0 .
$$

From Eq. (A1), solution (58) for integer values $\alpha=n, n=$ $1,2,3, \ldots$, can be expressed in terms of elementary functions:

$$
\begin{aligned}
\mu & =-\frac{1+\gamma}{n}<0, \quad-\infty<t<\infty, \\
f(r, t) & =\frac{(1+\gamma)\left|j_{0}\right| \tau_{0}}{r}(n-1) !(-t v)^{-n}\left[e^{-t v}-\sum_{k=0}^{n-1} \frac{(-t v)^{k}}{k !}\right], \\
n & =1,2 \ldots, \quad v=c r^{\mu}, \\
j & =-\frac{\left|j_{0}\right| \tau_{0}}{t}\left(1-n !(-t v)^{-n}\left[e^{-t v}-\sum_{k=0}^{n-1} \frac{(-t v)^{k}}{k !}\right]\right), \\
j & <0 .
\end{aligned}
$$

Using Eq. (62), $x=\frac{1}{3}, \mu=-\frac{2}{3}, \gamma=1, \alpha=3$, the expressions (65) can be applied to the problem of decaying turbulence:

$$
\begin{aligned}
\mu & =-\frac{2}{3}<0, \quad \gamma=1, \quad \alpha=3, \quad-\infty<t<\infty, \\
f(r, t) & =\frac{2 \varepsilon(t)}{r v}\left(1-\frac{2}{v t}+\frac{2}{(v t)^{2}}-\frac{2 e^{-v t}}{(v t)^{2}}\right), \\
v(r) & =\varepsilon_{0}^{1 / 3} r^{-2 / 3}, \\
j(r, t) & =-\varepsilon(t)\left[1-\frac{3}{v t}\left(1-\frac{2}{v t}+\frac{2}{(v t)^{2}}-\frac{2 e^{-v t}}{(v t)^{2}}\right)\right], \\
j & <0, \quad \varepsilon(t)=\frac{\varepsilon_{0} \tau_{0}}{t}, \quad \varepsilon_{0}=\varepsilon\left(\tau_{0}\right)=\left|j_{0}\right| .
\end{aligned}
$$

Under the time translation $t \rightarrow t+\tau_{0}$ in Eq. (66), and setting $\tau_{0} \rightarrow \infty$, the following stationary solution takes place:

$$
f(r)=2 \varepsilon_{0}^{2 / 3} r^{-1 / 3}, \quad j=-\varepsilon_{0}<0 .
$$

Then the distribution of turbulent energy density in size space can be expressed by the second-order longitudinal velocity structure function $D_{\|}(r)=\left\langle\left[\boldsymbol{v}_{\|}(\boldsymbol{x}+\boldsymbol{r})-\boldsymbol{v}_{\|}(\mathbf{x})\right]^{2}\right\rangle_{x}$ :

$$
\begin{aligned}
f(r) & =\frac{3}{2} \frac{\partial}{\partial r}\left\langle\left[\boldsymbol{v}_{\|}(\boldsymbol{x}+\boldsymbol{r})-\boldsymbol{v}_{\|}(\mathbf{x})\right]^{2}\right\rangle_{\boldsymbol{x}} \\
& =\frac{3}{2} \frac{\partial}{\partial r} C_{2} \varepsilon_{0}^{2 / 3} r^{2 / 3}=C_{2} \varepsilon_{0}^{2 / 3} r^{-1 / 3}, \\
\int_{0}^{\infty} f(r) d r & =3\left\langle\boldsymbol{v}_{\|}^{2}\right\rangle=\left\langle\boldsymbol{v}^{2}\right\rangle, \quad \boldsymbol{v}_{\|}=\frac{\boldsymbol{r}}{r^{2}} \boldsymbol{r} \cdot \boldsymbol{v}, \\
\left\langle\boldsymbol{v}^{2}\right\rangle & =3\left\langle\boldsymbol{v}_{\|}^{2}\right\rangle, \quad j(r)=-\varepsilon_{0}<0 .
\end{aligned}
$$

This distribution corresponds to the spectrum proposed by Kolmogorov for stationary homogeneous turbulence [16]. Remarkably, the solution (67) to the fragmentation equation (23) agrees with the Kolmogorov spectrum (68) even including the universal constant $\left(C_{2}=2\right.$ was established by measurements; see [17], for example). 


\section{Application to impact fragmentation of brittle solids}

Solution (58) has also application to the description of the impact fragmentation of brittle solids. The total mass is conserved during the impact fragmentation, and size of a fragment is commonly characterized by its volume. So, in this example, $r$ means the volume of the fragment, and $f(r) d r$ is the mass of fragments in the interval $d r$. Fragments of the impact fragmentation are generated by induced sound waves. If $c_{s}$ is the sound speed, then the time of the passage of sound waves across a fragment is $r^{1 / 3} / c_{s}$ and the fragmentation frequency $v(r)$ can be considered inversely proportional to this time. Thereby the fragmentation frequency depends on fragment's volume as $v(r) \sim r^{-1 / 3}$. In the framework of the cascade model of fragmentation, we have $\mu=-1 / 3$ in Eq. (5). The long-time limit (A5) of the mass distribution of fragments (58) in the volume interval $0<r<\infty$ has the following form:

$$
f(r) \sim \frac{1}{v r} \sim r^{-2 / 3} .
$$

Here the exponent in power law $-2 / 3$ is independent of material properties (of the sound speed in the considered case). The same universal exponent was found experimentally in Refs. [18,19], and also in studies referenced herein.

\section{The fragmentation rate is independent of fragment's size; $\mu=0$}

\section{Positive lognormal intermediate asymptotics; nonpositive asymptotic solutions}

Here we will consider the fragmentation equation in the form given by (18):

$$
\begin{aligned}
\frac{\partial}{\partial t} f & =\frac{\partial}{\partial r} r\left(\langle-\ln \alpha\rangle+\frac{1}{2}\left\langle(\ln \alpha)^{2}\right\rangle \frac{\partial}{\partial r} r \hat{I}_{+}^{(2)}\right) v f, \\
v & =c-\text { const. }
\end{aligned}
$$

For the sake of simplicity, we will use the logarithmic variable $w=\ln \left(\frac{r}{r_{0}}\right)$, where $r_{0}$ is an arbitrary constant. To the scaling transformation with generator $\hat{\sigma}=\frac{\partial}{\partial r} r$, we add the logarithmic scaling transformation with generator $\hat{\sigma} \ln \left(\frac{r}{r_{0}}\right)$. Generators $\hat{\sigma}$ and $\hat{\sigma} w$ induce Lie algebra. Their commutator is

$[\hat{\sigma} w, \hat{\sigma}]=-\hat{\sigma}, \quad \hat{\sigma}=\frac{\partial}{\partial r} r, \quad w=\ln \left(\frac{r}{r_{0}}\right), \quad r_{0}-$ const.

Scaling and logarithmic scaling transformations constitute a group, and the scaling transformations in this group are an invariant subgroup. The group generators can be expressed by logarithmic derivatives, and vice versa, as

$$
\begin{aligned}
& \hat{\sigma}=r^{-1} \frac{\partial}{\partial w} r, \quad \hat{\sigma} w=r^{-1} \frac{\partial}{\partial w} w r, \\
& \frac{\partial}{\partial w}=r \hat{\sigma} r^{-1}, \quad \frac{\partial}{\partial w} w=r \hat{\sigma} w r^{-1} \text {. }
\end{aligned}
$$

From Eq. (71), based on the well-known relation $e^{\hat{A}} \hat{B} e^{-\hat{A}}=$ $\sum_{n=0}^{\infty} \frac{1}{n !}[\hat{A},[\hat{A},[\hat{A}, \ldots \hat{B}] \ldots]]_{n}$, we have expressions for the $\hat{\sigma}$ generator as well as for the scaling operator $e^{(\ln \alpha) \hat{\sigma}}$ transformations under the similarity transformation induced by the logarithmic scaling operator $e^{(\ln b) \hat{\sigma} w}$ :

$$
\begin{gathered}
e^{(\ln b) \hat{\sigma} w} \hat{\sigma} e^{-(\ln b) \hat{\sigma} w}=b^{-1} \hat{\sigma}, \\
e^{(\ln b) \hat{\sigma} w} e^{(\ln \alpha) \hat{\sigma}} e^{-(\ln b) \hat{\sigma} w}=e^{b^{-1}(\ln \alpha) \hat{\sigma}} .
\end{gathered}
$$

It turns out that under these transformations, the fragmentation operator $\hat{I}_{+}$preserves the same form; only its fragmentation spectrum changes, $q(\alpha) \rightarrow q^{\prime}(\alpha)$ :

$$
e^{(\ln b) \hat{\sigma} w} \hat{I}_{+} e^{-(\ln b) \hat{\sigma} w}=\hat{I}_{+}^{\prime}, \quad q^{\prime}(\alpha)=b \alpha^{b-1} q\left(\alpha^{b}\right) .
$$

Note that if the parameter $b$ tends to infinity, the fragmentation spectrum $q^{\prime}(\alpha)$ tends to a $\delta$ function, and consequently, the fragmentation operator $\hat{I}_{+}^{\prime}$ tends to the unit operator:

$$
q^{\prime}(\alpha) \underset{b \rightarrow \infty}{\rightarrow} \delta(\alpha-1), \quad \hat{I}_{+}^{\prime} \underset{b \rightarrow \infty}{\rightarrow} 1
$$

In order to construct the self-similar solution to Eq. (18), we use the composition of scaling and logarithmic scaling transformations $\hat{L}$ :

$$
\begin{aligned}
\phi(r, t)= & \hat{L} f(r, t)=e^{(\ln \sqrt{v t}) \hat{\sigma} w} e^{\langle\ln \alpha\rangle v \hat{\sigma}} f(r, t) \\
f(r, t)= & \hat{L}^{-1} \phi(r, t)=e^{-\langle\ln \alpha\rangle v t \hat{\sigma}} e^{-(\ln \sqrt{v t}) \hat{\sigma} w} \phi(r, t) \\
= & r^{-1} \exp \left(-\langle\ln \alpha\rangle v t \frac{\partial}{\partial w}\right) \\
& \times \exp \left[-(\ln \sqrt{v t}) \frac{\partial}{\partial w} w\right] r \phi(r, t), \\
r \hat{L}^{-1} r^{-1}= & \exp \left(-\langle\ln \alpha\rangle v t \frac{\partial}{\partial w}\right) \exp \left[-(\ln \sqrt{v} t) \frac{\partial}{\partial w} w\right] \\
r \hat{L} r^{-1}= & \exp \left[(\ln \sqrt{v t}) \frac{\partial}{\partial w} w\right] \exp \left(\langle\ln \alpha\rangle v t \frac{\partial}{\partial w}\right) .
\end{aligned}
$$

The last two lines in Eq. (76) was obtained using Eq. (72). Under these transformations, the time derivative operator $\frac{\partial}{\partial t}$ and the operator $\hat{\sigma}$ transform as follows:

$$
\hat{L} \hat{\sigma} \hat{L}^{-1}=\frac{\hat{\sigma}}{\sqrt{v t}}, \quad \hat{L} \frac{\partial}{\partial t} \hat{L}^{-1}=\frac{\partial}{\partial t}-\frac{1}{2 t} \hat{\sigma} w-\frac{\langle\ln \alpha\rangle}{\sqrt{v t}} \nu \hat{\sigma} .
$$

Applying the operator $\hat{L}$ to Eq. (70), and using Eqs. (73)-(77), we obtain the equation for $\phi(r, t)$ :

$$
\frac{\partial}{\partial t} \phi=\frac{1}{2 t} \hat{\sigma}\left[\left\langle(\ln \alpha)^{2}\right\rangle \hat{\sigma} \hat{I}_{+}^{\prime}+w\right] \phi .
$$

In the limit $t \rightarrow \infty$, the function $\phi$ becomes independent of time, since the operator $\hat{I}_{+}^{\prime}=\hat{L} \hat{I}_{+}^{(2)} \hat{L}^{-1}$ degenerates to the unit operator, as shown by Eq. (75), and so it satisfies the following equation:

$$
\begin{aligned}
& \hat{\sigma}\left(\left\langle(\ln \alpha)^{2}\right\rangle \hat{\sigma}+w\right) \phi(r) \\
& \quad \equiv r^{-1} \frac{\partial}{\partial w}\left(\left\langle(\ln \alpha)^{2}\right\rangle \frac{\partial}{\partial w}+w\right) r \phi(r)=0 .
\end{aligned}
$$

This equation is equivalent to the simpler equation

$$
\left(\left\langle(\ln \alpha)^{2}\right\rangle \frac{\partial}{\partial w}+w\right) r \phi(r)=j_{0} .
$$

When $j_{0}=0$, the solution to this equation is the lognormal distribution:

$$
r \phi(r)=\frac{1}{\sqrt{2 \pi\left\langle(\ln \alpha)^{2}\right\rangle}} \exp \left[-\frac{w^{2}}{2\left\langle(\ln \alpha)^{2}\right\rangle}\right], \quad j_{0}=0 .
$$


If the bare mass flux is nonzero $\left(j_{0} \neq 0\right)$, the solution is given by

$$
\begin{aligned}
r \phi(r)= & \frac{j_{0}}{\left\langle(\ln \alpha)^{2}\right\rangle} \exp \left[-\frac{w^{2}}{2\left\langle(\ln \alpha)^{2}\right\rangle}\right] \\
& \times \int_{w_{0}}^{w} \exp \left[\frac{w^{2}}{2\left\langle(\ln \alpha)^{2}\right\rangle}\right] d w, \quad w_{0}-\text { const },
\end{aligned}
$$

There is no solution to Eq. (80) that is positive for all sizes $(-\infty<w<\infty)$ because the integral in Eq. (82) diverges for $w_{0}= \pm \infty$. Previously, in this paper, we did not analyze the nonpositive solutions. However, such solutions may have some physical sense when they are applied to the description of the density evolution of various nonpositive quantities. At $w_{0}=0$, solution (82) can be expressed using the imaginary error function erfi $(x)$. This solution has the same asymptotic behavior in regions of both small and large sizes:

$$
\begin{aligned}
& r \phi(r)= \frac{j_{0}}{\left\langle(\ln \alpha)^{2}\right\rangle} \exp \left[-\frac{w^{2}}{2\left\langle(\ln \alpha)^{2}\right\rangle}\right] \\
& \times \int_{0}^{w} \exp \left[\frac{w^{2}}{2\left\langle(\ln \alpha)^{2}\right\rangle}\right] d w \\
&= j_{0} \sqrt{\frac{\pi}{2\left\langle(\ln \alpha)^{2}\right\rangle}} \exp \left[-\frac{w^{2}}{2\left\langle(\ln \alpha)^{2}\right\rangle}\right] \\
& \times \operatorname{erfi}\left(\frac{w}{\sqrt{2\left\langle(\ln \alpha)^{2}\right\rangle}}\right) \\
& \phi(r) \underset{\ln (r) \rightarrow \pm \infty}{\rightarrow} \frac{j_{0}}{r \ln (r)} .
\end{aligned}
$$

Applying the operator $\hat{L}^{-1}$ from Eq. (76) to the function $\phi(r)$ given by Eq. (83), we obtain the self-similar nonpositive solution to Eq. (70):

$$
\begin{aligned}
& f(r, t)= j_{0} r^{-1} \sqrt{\frac{\pi}{2\left\langle(\ln \alpha)^{2}\right\rangle \nu t}} \exp \left[-\frac{(w-\langle\ln \alpha\rangle v t)^{2}}{2\left\langle(\ln \alpha)^{2}\right\rangle \nu t}\right] \\
& \times \operatorname{erfi}\left(\frac{w-\langle\ln \alpha\rangle v t}{\sqrt{2\left\langle(\ln \alpha)^{2}\right\rangle \nu t}}\right), \\
& f(r, t) \underset{\ln (r) \rightarrow \pm \infty}{\sim} j_{0} r^{-1} \frac{1}{\ln (r)-\langle\ln \alpha\rangle v t}, \quad\langle\ln \alpha\rangle<0 .
\end{aligned}
$$

There is an interesting application of this result in twodimensional turbulence [20].

\section{Application to two-dimensional turbulence}

For a two-dimensional inviscid flow, the vertical component of vorticity $\omega=[\nabla \times \boldsymbol{v}]_{z}$ is conserved, as are all its powers $\omega^{n}$. Therefore again, instead of mass distribution in the size space, we consider the density of the third-order binomial of vorticity $\left\langle\omega^{3}\right\rangle-\langle\omega\rangle\left\langle\omega^{2}\right\rangle$ in size space. Then this density $f(r, t)$ and the flux will have the dimensions $[f]=\left[\frac{v^{3}}{r^{4}}\right],\left[j_{0}\right]=\left[\frac{v^{4}}{r^{4}}\right]$, respectively. Consequently, assuming universality (60) of the fragmentation rate constant $c$, we have

$$
x=\frac{1}{4}, \quad \mu=0 .
$$

With these exponents, the distribution function $f(r, t)$ in Eq. (84) can be interpreted as density of $\left[\left\langle\omega^{3}\right\rangle-\langle\omega\rangle\left\langle\omega^{2}\right\rangle\right]$ in size space in two-dimensional turbulence:

$$
\begin{aligned}
f(r, t) & =\frac{\partial}{\partial r}\left\langle[\omega(\boldsymbol{x}+\boldsymbol{r}, t)-\omega(\boldsymbol{x}, t)][\omega(\boldsymbol{x}+\boldsymbol{r}, t)]^{2}\right\rangle_{\boldsymbol{x}}, \\
\int_{0}^{\infty} f(r, t) d r & =\left\langle\omega^{3}\right\rangle-\langle\omega\rangle\left\langle\omega^{2}\right\rangle, \\
v & =c=\left|j_{0}\right|^{1 / 4} .
\end{aligned}
$$

Finally, the following result can be derived for the third vorticity moment size density in the two dimensions:

$$
\begin{gathered}
\frac{\partial}{\partial r}\left\langle[\omega(\boldsymbol{x}+\boldsymbol{r}, t)-\omega(\boldsymbol{x}, t)][\omega(\boldsymbol{x}+\boldsymbol{r}, t)]^{2}\right\rangle_{\boldsymbol{x}} \\
=j_{0} r^{-1} \sqrt{\frac{\pi}{2\left\langle(\ln \alpha)^{2}\right\rangle \nu t}} \exp \left[-\frac{[\ln (r)-\langle\ln \alpha\rangle \nu t]^{2}}{2\left\langle(\ln \alpha)^{2}\right\rangle \nu t}\right] \\
\quad \times \operatorname{erfi}\left(\frac{\ln (r)-\langle\ln \alpha\rangle v t}{\sqrt{2\left\langle(\ln \alpha)^{2}\right\rangle v t}}\right), \\
\frac{\partial}{\partial r}\left\langle[\omega(\boldsymbol{x}+\boldsymbol{r}, t)-\omega(\boldsymbol{x}, t)][\omega(\boldsymbol{x}+\boldsymbol{r}, t)]^{2}\right\rangle_{\boldsymbol{x}} \\
\underset{\ln (r) \rightarrow \pm \infty}{\sim} j_{0} r^{-1} \frac{1}{\ln (r)-\langle\ln \alpha\rangle \nu t}, \\
v=\left|j_{0}\right|^{1 / 4}=\mathrm{const}, \quad\langle\ln \alpha\rangle<0 .
\end{gathered}
$$

The positive solution to Eq. (80) is the lognormal distribution (81), and the "bare" flux should be zero $\left(j_{0}=0\right)$. From this, in accordance with the second line of Eq. (76), we finally obtain the well-known lognormal asymptotic behavior at large times for the fragmentation equation (3) with a constant fragmentation rate:

$$
\begin{aligned}
f(r, t) \underset{t \rightarrow \infty}{\sim} r^{-1} & \exp \left(-\langle\ln \alpha\rangle v t \frac{\partial}{\partial w}\right) \exp \left(-\ln \sqrt{v t} \frac{\partial}{\partial w} w\right) \\
& \times \frac{1}{\sqrt{2 \pi\left\langle(\ln \alpha)^{2}\right\rangle}} \exp \left[-\frac{w^{2}}{2\left\langle(\ln \alpha)^{2}\right\rangle}\right] \\
= & r^{-1} \frac{1}{\sqrt{2 \pi\left\langle(\ln \alpha)^{2}\right\rangle \nu t}} \exp \left[-\frac{(w-\langle\ln \alpha\rangle v t)^{2}}{2\left\langle(\ln \alpha)^{2}\right\rangle v t}\right], \\
v= & \text { const. }
\end{aligned}
$$

Notice that replacing the operator $\hat{I}_{+}^{\prime}$ by unity in Eq. (78) for $\phi(r, t)$ at large times is equivalent to replacing the operator $\hat{I}_{+}^{(2)}$ by unity in the primary equation (18) for $f(r, t)$. From this, we can conclude that evolution of the fragmentation process under scaling symmetry on large times is equivalently governed by the Fokker-Planck type equation:

$$
\begin{aligned}
\frac{\partial}{\partial t} f & =\frac{\partial}{\partial r} r\left(\langle-\ln \alpha\rangle+\frac{1}{2}\left\langle(\ln \alpha)^{2}\right\rangle \frac{\partial}{\partial r} r\right) v f, \\
v & =\text { const }, \quad t \rightarrow \infty .
\end{aligned}
$$

It is also remarkable that as time progresses even further, the lognormal distribution (88) becomes a power one with an exponent independent of the initial distribution.

$$
\begin{aligned}
f(r, t) \underset{t \rightarrow \infty}{\sim} & \frac{1}{r_{0}} \frac{\exp \left[-(\langle\ln \alpha\rangle)^{2} v t /\left\langle(\ln \alpha)^{2}\right\rangle\right]}{\sqrt{2 \pi\left\langle(\ln \alpha)^{2}\right\rangle \nu t}} \\
& \times\left(\frac{r_{0}}{r}\right)^{1-\langle\ln \alpha\rangle /\left\langle(\ln \alpha)^{2}\right\rangle} .
\end{aligned}
$$


Recalling that Eq. (70) also has a stationary solution (for $0<r<\infty$ ) [see Eq. (24)]:

$$
\begin{aligned}
f(r, t) & =-j_{0}\left(\frac{1}{\langle-\ln \alpha\rangle \nu r}+t \delta(r)\right), \\
j_{0} & <0 ; \quad v-\mathrm{const} ; \quad 0 \leqslant r<\infty .
\end{aligned}
$$

In this case, the distribution function decreases inversely with the radius, $f(r) \sim r^{-1}$. This distribution provides a steady mass flux $j_{0}$, which is directed towards smaller scales. If now instead of the mass distribution, we consider the density of the second-order binomial of vorticity $\left\langle\omega^{2}\right\rangle-\langle\omega\rangle^{2}$ in size space; then this density $f(r, t)$ and its flux will have the following dimensions: $[f]=\frac{v^{2}}{r^{3}},[j]=\frac{v^{2}}{r^{3}} v=\frac{1}{t^{3}}$. For this case, from the universality of the fragmentation constant $c$ (61), we have

$$
x=\frac{1}{3}, \quad \mu=0 .
$$

With these exponents, solution (91) can provide a description of the enstrophy spectrum for two-dimensional steady turbulence. For this case, the flux towards the zero size $j_{0}$ can be identified with the specific dissipation of enstrophy, denoted by $\eta$ :

$$
\eta=-j(r=0)=\left|j_{0}\right| .
$$

So the distribution of the specific enstrophy behaves inversely with size. Finally, this distribution is

$$
\begin{aligned}
f(r) & =\frac{1}{2} \frac{\partial}{\partial r}\left\langle[\omega(\boldsymbol{x}+\boldsymbol{r})-\omega(\boldsymbol{x})]^{2}\right\rangle_{x}=\frac{\eta^{2 / 3}}{\langle-\ln \alpha\rangle} \frac{1}{r}, \\
0 & <r<\infty .
\end{aligned}
$$

\section{CONCLUSION}

In this paper, the approach [13] for renormalization of the Boltzmann collision integral is used to obtain the renormalized form of the fragmentation operator. This allows the fragmentation equation to be rewritten as the continuity equation in size space. An advantage of this exact conversion of the primary integro-differential equation to its continuity form is as follows. The primary fragmentation equation provides a statistical description of the temporal evolution of fragmenting particles; in size space, each such particle jumps, but has no velocity. On the contrary, the renormalized fragmentation equation describes evolution in time of quasiparticles which move at a certain velocity in size space, and this velocity depends on the positions of all the other quasiparticles. The renormalization of the fragmentation equation provides an example of a statistically equivalent description, in which the microdynamics can be substantially modified without changing the distribution function. The renormalized fragmentation equation contains an explicit expression for the mass flux. This allowed us to derive an integral of motion for self-similar solutions. This integral is referred to as the bare flux. When the breakup frequency is a power function of size, a simple exact stationary solution is obtained, providing a stationary mass flux, from infinity towards zero size. On the basis of the continuity form of the fragmentation equation and specifically introduced scaling transformations, we have obtained and analyzed self-similar solutions. Three physically different situations have been considered: With decreasing the size, the fragmentation rate can either decrease, increase, or remain constant. The obtained solutions include solutions with an infinite norm, and also solutions which are nonpositive. We have provided a possible interpretation of such solutions. We have proposed an empirico-mathematical model for a turbulent cascade which mimics the main process in hydrodynamic turbulence: "A piece of swirling fluid produces additional smaller swirls." This model consists of the following steps. Using the divergence form of the fragmentation equation, and working in size space, we can associate the flux towards zero size ("condensation" to zero size) with the dissipation rate either of turbulent energy in the case of 3D decaying turbulence or of the vorticity moments in the case of $2 \mathrm{D}$ turbulence. We linked the fragmentation rate to the bare flux through a universality condition (60), and we obtained a set of exact self-similar solutions. The universal asymptotics (66) and (67) describe the relaxation of density of specific turbulent energy in size space in homogeneous isotropic turbulence. The limiting forms of these asymptotics agree with the Kolmogorov stationary spectrum, even including the value of the constant, which is usually presented in turbulence as an empirical one. The nonpositive self-similar solution (87) at constant fragmentation rate is attributed to relaxation of the density of the vorticity moments in size space in two-dimensional decaying turbulence. Alternatively, the positive solution in this case is linked to the spectrum of enstrophy according to (94). It would be interesting to verify the predicted spectra in measurements. We also suggested a simple empirical cascade model of impact fragmentation of brittle materials which gives the correct value of the universal power law exponent of fragment sizes and masses.

\section{APPENDIX}

The integral in Eq. (41) can be expressed by confluent hypergeometric functions [15] $\Phi(1,1+\alpha ; x)$ and $\Psi(1,1+$ $\alpha ; x)$; if $\alpha$ is an integer, then $\Phi(1,1+\alpha ; x)$ is the elementary function

$$
\begin{aligned}
\int_{0}^{\nu} x^{\alpha-1} e^{\tau x} d x & =\alpha^{-1} \nu^{\alpha} e^{\tau \nu} \Phi(1,1+\alpha,-\tau \nu), \quad \operatorname{Re} \alpha>0, \\
\int_{\nu}^{\infty} x^{\alpha-1} e^{\tau x} d x & =v^{\alpha} e^{\tau \nu} \Psi(1,1+\alpha,-\tau \nu), \quad \operatorname{Re} \tau<0, \\
v & >0, \quad \Phi(1,1+n, x)=n ! x^{-n}\left[e^{x}-\sum_{k=0}^{n-1} \frac{x^{k}}{k !}\right], \\
n & =0,1, \ldots ; \\
\Phi(1,1+\alpha, x) & =e^{x} \Phi(\alpha, 1+\alpha,-x) .
\end{aligned}
$$

The asymptotic behavior of confluent hypergeometric functions $\Phi(1,1+\alpha ; x)$ and $\Psi(1,1+\alpha ; x)$ is given in Ref. [15]:

$$
\begin{aligned}
& \Phi(1,1+\alpha, x) \underset{x \rightarrow-\infty}{\rightarrow} \alpha(-x)^{-1}, \\
& \Phi(1,1+\alpha, x) \underset{x \rightarrow 0}{\rightarrow} 1, \\
& \Phi(1,1+\alpha, x) \underset{x \rightarrow \infty}{\sim} \alpha x^{-\alpha} e^{x},
\end{aligned}
$$




$$
\begin{aligned}
& \Psi(1,1+\alpha, x) \underset{x \rightarrow 0}{\sim} \begin{cases}(-\alpha)^{-1}, & \alpha<0 ; \\
\Gamma(\alpha) x^{-\alpha}, & \alpha>0 ;\end{cases} \\
& \Psi(1,1+\alpha, x) \underset{x \rightarrow \infty}{\sim} x^{-1} .
\end{aligned}
$$

For these functions, there are two convenient integral representations [15]:

$$
\begin{aligned}
\Phi(a, b, x) & =\frac{\Gamma(b)}{\Gamma(b-a) \Gamma(a)} \int_{0}^{1} e^{x t} t^{a-1}(1-t)^{b-a-1} d t, \\
\Psi(a, b, x) & =\int_{0}^{\infty} e^{-x t} t^{a-1}(1+t)^{b-a-1} d t, \\
\operatorname{Re} x & >0, \quad \operatorname{Re} a>0 .
\end{aligned}
$$

For small and large sizes, solutions (49) have the following asymptotic behavior:

$$
\begin{aligned}
& 0<\mu, \quad \alpha<0, \quad j_{0}<0, \quad f(r, t) \underset{r \rightarrow 0}{\sim}-\frac{j_{0} \mu \tau_{0}}{r}, \\
& f(r, t) \underset{r \rightarrow \infty}{\sim} \frac{(1+\gamma) j_{0} \tau_{0}}{r v t}, \quad-\infty<t<0, \\
& f(r, t) \underset{r \rightarrow 0}{\sim} \operatorname{const}_{1}\left(\tau_{0}^{-1} t\right)^{-\alpha} r^{\gamma}, \\
& f(r, t) \underset{r \rightarrow \infty}{\rightarrow} 0, \quad 0<t<\infty .
\end{aligned}
$$

Solution (58) has the following asymptotic behavior in time:

$$
\begin{aligned}
& \mu<0, \quad f(r, t) \underset{t \rightarrow-\infty}{\sim} \frac{(1+\gamma)\left|j_{0}\right| \tau_{0}}{r}(-v t)^{-\alpha} e^{-v t}, \\
& j \underset{t \rightarrow-\infty}{\sim}-\frac{\left|j_{0}\right| \tau_{0}}{t} \alpha(-t \nu)^{-\alpha} e^{-t v} \underset{t \rightarrow-\infty}{\rightarrow}-\infty,
\end{aligned}
$$

[1] A. N. Kolmogorov, Dokl. Akad. Nauk. SSSR 31, 99 (1941).

[2] A. F. Filippov, Theory Probab. Appl. 4, 275 (1961).

[3] M. A. Gorokhovski and V. L. Saveliev, J. Phys. D: Appl. Phys. 41, 085405 (2008).

[4] R. M. Ziff and E. D. McGrady, J. Phys. A: Math. Gen. 18, 3027 (1985).

[5] E. D. McGrady and R. M. Ziff, Phys. Rev. Lett. 58, 892 (1987).

[6] R. M. Ziff, J. Phys. A: Math. Gen. 24, 2821 (1991).

[7] Z. Cheng and S. Redner, Phys. Rev. Lett. 60, 2450 (1988).

[8] Z. Cheng and S. Redner, J. Phys. A: Math. Gen. 23, 1233 (1990).

[9] A. A. Kostritsa and V. L. Saveliev, Izv. Akad. Nauk Kaz. SSR, Ser. Fiz. Mat. 6, 65 (1976).

[10] J. Bertoin, Random Fragmentation and Coagulation Processes (Cambridge University Press, Cambridge, 2006).

[11] B. Haas, Ann. Appl. Probab. 20, 382 (2010).

$$
\begin{aligned}
& \lim _{t \rightarrow 0} f(r, t)=-\frac{\mu\left|j_{0}\right| \tau_{0}}{r}, \quad j \underset{t \rightarrow 0}{\sim}-\left|j_{0}\right| \tau_{0} \frac{v}{1+\alpha}, \\
& f(r, t) \underset{t \rightarrow \infty}{\sim} \frac{(1+\gamma)\left|j_{0}\right| \tau_{0}}{r v t}, \\
& j \underset{t \rightarrow \infty}{\sim}-\frac{\left|j_{0}\right| \tau_{0}}{t}\left[1-\alpha(t v)^{-1}\right] \underset{t \rightarrow \infty}{\rightarrow}-\frac{\left|j_{0}\right| \tau_{0}}{t} .
\end{aligned}
$$

For small and large sizes, asymptotic expressions for the distribution function and the mass flux (58) are, respectively,

$$
\begin{aligned}
& \mu<0,-\infty<t<0, \\
& f(r, t) \underset{r \rightarrow 0}{\sim} \frac{(1+\gamma)\left|j_{0}\right| \tau_{0}}{r}(-t c)^{-\alpha} r^{\gamma+1} e^{-t c r^{\mu}} \underset{r \rightarrow 0}{\rightarrow} \infty, \\
& f(r, t) \underset{r \rightarrow \infty}{\sim}-\frac{\mu\left|j_{0}\right| \tau_{0}}{r}, \\
& j \underset{r \rightarrow 0}{\sim}-\frac{\left|j_{0}\right| \tau_{0}}{t} \alpha(-t v)^{-\alpha} e^{-t \nu} \underset{r \rightarrow 0}{\rightarrow}-\infty, \\
& j \underset{r \rightarrow \infty}{\sim}-\left|j_{0}\right| \tau_{0} \frac{c r^{\mu}}{1+\alpha} \underset{r \rightarrow \infty}{\rightarrow} 0, \\
& 0<j_{0} ; \quad \mu<0, \quad 0<t<\infty, \\
& f(r, t) \underset{r \rightarrow 0}{\sim} \frac{(1+\gamma)\left|j_{0}\right| \tau_{0}}{r \nu t}=\frac{(1+\gamma)\left|j_{0}\right| \tau_{0}}{c t} r^{-(\mu+1)}, \\
& f(r, t) \underset{r \rightarrow \infty}{\sim} \frac{\mu\left|j_{0}\right| \tau_{0}}{r}, \\
& j \underset{r \rightarrow 0}{\sim}-\frac{\left|j_{0}\right| \tau_{0}}{t}\left[1-\alpha(t v)^{-1}\right] \underset{r \rightarrow 0}{\rightarrow}-\frac{\left|j_{0}\right| \tau_{0}}{t}, \\
& j \underset{r \rightarrow \infty}{\sim}-\left|j_{0}\right| \tau_{0} \frac{c r^{\mu}}{1+\alpha} \underset{r \rightarrow \infty}{\rightarrow} 0, \quad j_{0}<0 .
\end{aligned}
$$

[12] J. Banasiak and S. C. Oukoumi Noutchie, Physica D 239, 1422 (2010).

[13] V. L. Saveliev and K. Nanbu, Phys. Rev. E 65, 051205 (2002).

[14] V. L. Saveliev and M. A. Gorokhovski, Phys. Rev. E 72, 016302 (2005).

[15] H. Bateman, Higher Transcendental Functions (Mcgraw-Hill Book Company, Inc., New York, 1953).

[16] A. S. Monin and A. M. Yaglom, Statistical Fluid Mechanics: Mechanics of Turbulence, Vol. 2 (MIT Press, Cambridge, 1975).

[17] S. B. Pope, Turbulent Flows (Cambridge University Press, Cambridge, 2000).

[18] H. Inaoka and M. Ohno, Fractals 11, 369 (2003)

[19] L. Oddershede, P. Dimon, and J. Bohr, Phys. Rev. Lett. 71, 3107 (1993).

[20] R. H. Kraichnan and D. Montgomery, Rep. Prog. Phys. 43, 547 (1980). 